\title{
POTENCIAL ANTIOXIDANTE DE Lippia alba (MILL.) N.E. BR. OCORRENTE NA REGIÃO DO PONTAL DO TRIANGULO MINEIRO, MG.
}

\author{
Priscila Ferrari de Morais ${ }^{1}$ \\ Luciane Maria Aparecida Nogueira² \\ Juliana Aparecida Povh ${ }^{3}$
}

Resumo: O Cerrado inclui considerável variedade de fisionomias vegetais, devido à expansão agrícola muitas espécies nativas estão sendo perdidas de maneira irreversível. Plantas da família Verbenaceae ocorrem no Cerrado brasileiro e estão incluídas espécies aromáticas, e que, podem apresentar substâncias com potencial medicinal. No Brasil Lippia alba ocorre em praticamente todas as regiões, onde é muito utilizada como planta medicinal por suas propriedades sedativa, carminativa e analgésica. Os compostos fenólicos encontrados nas plantas possuem alto poder antioxidante. Os antioxidantes são compostos químicos que podem diminuir a ação de radicais livres no organismo. Desse modo, o presente trabalho tem por objetivo quantificar o potencial antioxidante em dois quimiotipos de Lippia alba. Os resultados demonstram valores significativamente maiores de fenóis totais, flavonoides e atividade antioxidante em L. alba 2 em relação à L. alba 1.

Palavras-chave: Cerrado; Quimiotipo; Óleos essenciais; Verbenaceae..

\footnotetext{
${ }^{1}$ Ciências Biológicas/Universidade Federal de Uberlândia (Faculdade de Ciências Integradas do Pontal), Brasil. E-mail: priferrari4@gmail.com.

2 Ciências Biológicas/Universidade Federal de Uberlândia (Faculdade de Ciências Integradas do Pontal), Brasil. E-mail: lulu_smile123@hotmail.com.

${ }^{3}$ Ciências Biológicas/Universidade Federal de Uberlândia (Faculdade de Ciências Integradas do Pontal), Brasil. E-mail: japovh@pontal.ufu.br.
} 